\title{
SENSO DE COERÊNCIA DE MÃES EM VULNERABILIDADE SOCIAL E O ESTADO NUTRICIONAL DE SEUS FILHOS: UMA REVISÃO
}

\author{
Diomar Maria do Nascimento Araújo ${ }^{1}$ \\ Islane Cristina Martins ${ }^{2}$
}

RESUMO: Introdução: $O$ senso de coerência (SOC) forte pode permitir que mães de baixa renda, consiga ser capaz de manter um peso saudável e um comportamento alimentar adequado de seus filhos, apesar dos recursos limitados e dos eventos estressantes da vida. Objetivo: Realizar uma revisão integrativa da literatura a fim de avaliar como se dá o senso de coerência de mães em vulnerabilidade social e o estado nutricional de seus filhos. Material e Métodos: Foi feito um levantamento da literatura no mês de março de 2021, nas bases de dados Periódicos CAPES, Google Scholar e Pubmed. Os descritores utilizados foram os seguintes: "Senso de Coerência” AND “Mães" AND "Alto Risco Social” AND 'Estado de Nutrição" e, em inglês, "Sense of Coherence" AND "Mothers" AND "High Social Risk" AND "Nutrition Status" em todas as bases de dados. Foram selecionados 04 artigos que se adequaram segundo os critérios de elegibilidade. Resultados: Foi possível observar que $100 \%$ dos artigos fizeram menção que mães com SOC forte eram propensas a praticar comportamentos saudáveis em seus filhos. Sendo unânime entre os artigos que nível alto senso de coerência está relacionado com baixo nível de estresse e que o apoio social (amigos e família) foi um fator positivo associado. A influência do SOC no status socioeconômico e na educação até então, existem divergências. Conclusão: Os artigos permitiram identificar evidências de que mães com senso de coerência forte eram predispostas a praticar comportamentos saudáveis e lidam melhor com as adversidades. Reforçam que a prevalência de pré-escolares com excesso peso, está relacionada com baixo SOC materno, porém estudos com essas variáveis são ainda escassos.

Palavras-chave: Senso de Coerência. Mães. Alto Risco Social. Estado de Nutrição.

ABSTRACT: Introduction: The strong sense of coherence (SOC) may allow that the low-income mothers to be able to maintain a healthy weight and adequate eating behavior for their children, despite limited resources and stressful life events. Objective: Carrying out an integrative literature review in order to assess how the sense of coherence of mothers in social vulnerability and the nutritional status of their children occurs. Materials and Methods: It was carried out a literature survey

\footnotetext{
${ }^{1}$ Nutricionista, pelo Centro Universitário Estácio do Recife. Especialização em Saúde Pública com Ênfase em Saúde da Família- Sanitarista pela Faculdade José Lacerda Filho Ciências AplicadasFAJOLCA.E-mail: diomarnutri@hotmail.com.

${ }^{2}$ Biomédica pela Universidade Federal de Pernambuco-PE, mestre em Neurociências pelo Programa de Pós-Graduação em Neuropsiquiatria e Ciências do Comportamento-UFPE e doutora pelo PPGBAS LIKA-UFPE. Professional and self coach-IBC. E-mail: islanemartins@gmail.com.
} 
in March 2021, in the CAPES, Google Scholar and Pubmed databases. The descriptors used were as follows: "Sense of Coherence "AND " Mothers' AND " High Social Risk" AND " State of Nutrition " and, in English, " Sense of Coherence "AND " Mothers" AND " High Social Risk "AND " Nutrition Status" in all databases. It was selected 04 articles that fit according to the eligibility criteria Results: It was possible to note that $100 \%$ of the articles mentioned that mothers with a strong SOC were prone to practice healthy behaviors in their children. Being unanimous among the articles that a high sense of coherence is associated to a low level of stress and that social support (friends and family) was an associated positive factor. SOC's influence on socioeconomic status and education until then, there are divergences. Conclusion: The articles allowed identifying evidence that mothers with a strong sense of coherence were predisposed to practice healthy behaviors and deal better with adversity. They reinforce that the prevalence of overweight preschoolers is related to low maternal SOC, however studies with these variables are still scarce.

Keywords: Sense of Coherence. Mothers. High Social Risk. Nutrition Status.

\section{INTRODUÇÃO}

A capacidade de um indivíduo de lidar com os eventos estressores ao longo da vida ocasionando resposta do organismo com diferentes reações, frente aos estímulos identificados foi elucidada anteriormente por meio do senso de coerência (SOC) (ANTONOVSKY, 1993).

Portanto, o SOC se forma por meio da interação entre recursos externos (baseado no ambiente) e internos (próprio da pessoa), criando um curso de vida coerente, gerenciável e estruturado, mesmo em condições desfavoráveis (BAUER et al.,2019).

Dito isso, uma das possíveis causas do excesso de peso em crianças em idade pré-escolar, seria o fraco senso de coerência materno. Esse fenômeno pode ocorrer devido ao estresse e as respostas que ele provoca (ELI et al.,2016).

Logo, um indivíduo com um SOC forte terá os estímulos estressantes previsíveis e compreensíveis, além de possuir os recursos para superar esses estímulos e, manter sua saúde. Assim sendo, a sugestão é de um constructo universal, encontrado em todos. (MIZUTA et al.,2020).

Não só isso, mas a forma como as crianças gastam energia tem implicações no peso corporal além da alimentação. Portanto, gastar tempo na exibição de telas como 
televisão e videogame foi associado ao aumento do risco de obesidade e do consumo de calorias (SPEIRS et al., 2016).

Embora, crianças de baixa renda sejam menos propensas a ter espaços compartilhados (por exemplo, parques e ciclovias) que promovam atividade física em suas comunidades e são mais propensas a experimentar altos níveis de estresse e eventos estressantes (SPEIRS et al., 2016).

No entanto, acredita-se que mães em vulnerabilidade social tendo um o senso de coerência aumentado lidar melhor com as adversidades, e se adaptam a situações de estresse, tendo qualidade de vida e comportamento alimentar saudável, repercutindo em seus filhos (SPEIRS et al., 2016).

Com isso, o empenho para avaliar o senso de coerência (SOC) materno em pré-escolares tem sido incentivo para pesquisas, pois estudos nessa área são escassos. E, mães que tinham um senso mais elevado de coerência eram menos propensas a se envolver em práticas alimentares restritas.

Salienta-se que, conhecer a realidade desse grupo populacional cria subsídios para direcionar estratégias mais adequadas de promoção de hábitos alimentares saudáveis e também conduzi novas intervenções na obesidade infantil e futuras pesquisas na área (ELI et al.,2016).

Por isso, o objetivo do presente estudo foi buscar evidências na literatura através da revisão integrativa, para reunir e sintetizar o conhecimento produzido sobre o Senso de coerência de mães e o estado nutricional de seus filhos.

\section{MATERIAIS E MÉTODOS}

Foi feito um levantamento da literatura no mês de março de 202I, nas bases de dados Periódicos CAPES, Google Scholar e Pubmed. Os descritores utilizados foram os seguintes: "Senso de Coerência" AND "Mães" AND 'Alto Risco Social” AND " Estado de Nutrição" e, em inglês, "Sense of Coherence" AND "Mothers" AND "High Social Risk" AND 'Nutrition Status" em todas as bases de dados. Foram selecionados 04 artigos sendo incluídos segundo os critérios de elegibilidade conforme a Figura I. Os critérios de inclusão foram: artigos nos idiomas inglês e português, nos últimos cinco anos, envolvendo o Senso de coerência, vulnerabilidade 
social e o estado nutricional de seus filhos. Os critérios de exclusão foram artigos de revisão de literatura ou metanálise.

FIGURA I. FLUXOGRAMA E CRITÉRIOS DE SELEÇÃO E INCLUSÃO DOS ARTIGOS
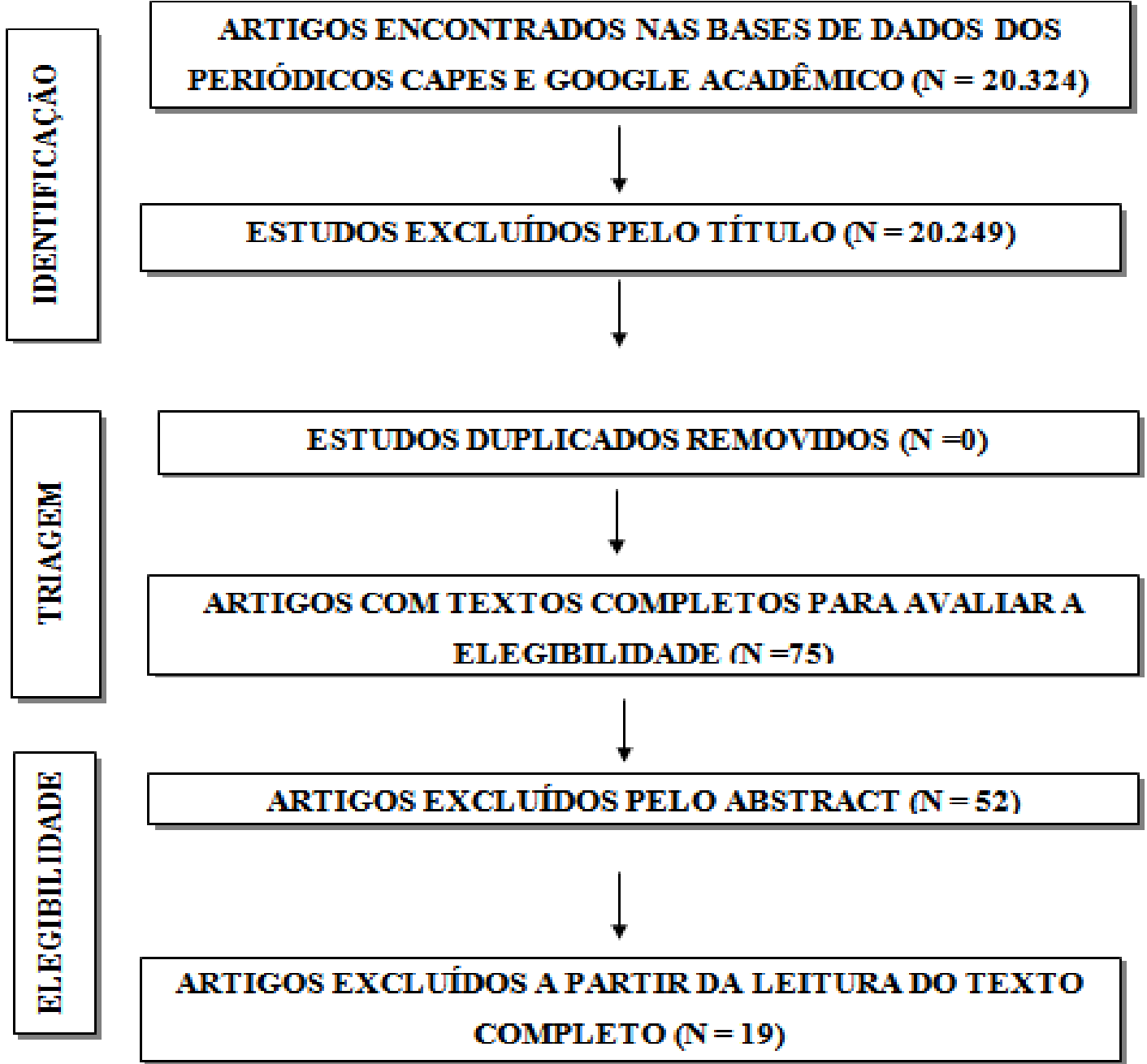

合

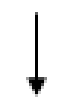

ESTUDOS INCLUÍDOS NA SÍNTESE QUALITATIVA (N=04)

\section{RESULTADOS}

Os resultados do presente estudo encontram-se no na Tabela I. 
Revista Ibero- Americana de Humanidades, Ciências e Educação- REASE

Tabela I - Demonstrativo dos artigos que integram a Revisão Integrativa

\#N Data

Título
Autores

Periódico

Perception of family sense of 2020 coherence during parental transition: A qualitative study.

Fei Wan Ngai e Pui Sze Chan

Journal of Health Psychology

First-TimeMothers'

Enjoyment of Breastfeeding Correlates with Duration of Agnes

Breastfeeding, Sense of Coherence, and Parental Ekstrem, Anette Couple and Child Relation: A Caroline Backstrom. Longitudinal Swedish Cohort Study.

Quality of couple relationship among first-time mothers and 2018 partners, during pregnancy and the first six months of parenthood.
Caroline Bäckström Ingemar Kåreholt, Sexu Stina Thorstenssona ,Marie Golsäterc Lena B. Mårtensson
Sexual Reproductive
Healthcare
Objetivos

Examinar o conceito de senso de coerência familiar e os fatores
Avaliar fatores associados à qualidade do relacionamento do casal, entre mães de primeira viagem e parceiros durante a gravidez $e$ os seis primeiros meses de paternidade.

\section{Resultados}

36 casais participaram do estudo. Vinte e dois casais (6o, $1 \%$ ) foram pais pela primeira vez. A média de idade das mães pela primeira e segunda vez foi de 32,8 anos $(D P=4,0)$ e 34,9 anos $(D P=3,3)$, respectivamente. A maioria dos casais tinha o ensino superior completo. Mais de 70 por cento das mulheres grávidas estavam empregadas e too por cento de seus parceiros estavam empregados. Os resultados semelhante do senso de coerência familiar no contexto da paternidade chinesa.

Investigar diversos fatores correlacionados com o prazer amamentação pelas mães de primeira viagem e a duração $d a$ amamentação, entre o parto dois anos após o nascimento.

99,2\% das mães iniciaram a amamentação após o nascimento. As frequências de amamentação foram $54,8 \%$ seis meses, $9,1 \%$ um ano e 1,0\% em dois anos. Maior grau em que as mães gostavam de amamentar e duração da amamentação foi correlacionada positivamente com um maior grau de prazer em amamentar, uma relação mais positiva com a criança, percepção mais forte relacionamento do casal parental e maior senso de coerência.

207 participantes foram elegíveis para análise (mães de primeira viagem $\mathrm{n}=122$; parceiros $n=85$ ). Os resultados do teste de Wilcoxon Signed Rank mostraram que a qualidade percebida do relacionamento do casal não mudou significativamente entre a gravidez $\left(\mathrm{Q}_{1}\right)$ e há primeira semana após o parto $\left(\mathrm{Q}_{2}\right)$. Houve uma diminuição na percepção da qualidade do relacionamento conjugal das mães pela primeira vez $(\mathrm{p}=\mathrm{o}, \mathrm{ooI})$ e dos parceiros $(\mathrm{p}<\mathrm{o}, \mathrm{ooI})$ entre a gravidez ( $\mathrm{Q}$ ) e seis meses após o nascimento $\left(\mathrm{Q}_{3}\right)$. Também houve diminuição entre há primeira semana após o parto $\left(\mathrm{Q}_{2}\right)$ e seis meses após o parto $\left(\mathrm{Q}_{3}\right)$, tanto entre as primíparas $(\mathrm{p}$ $<0,00 I)$ quanto entre os companheiros ( $\mathrm{p}<\mathrm{o}, \mathrm{ooI})$. Tanto a diminuição entre $\mathrm{Q}_{\mathrm{I}}$ e $\mathrm{Q}_{3}$ (mães pela primeira vez: $\eta_{2}=0,144$; parceiros: $\eta_{2}=0,365$ ) e entre $Q_{2}$ e $Q_{3}$ (mães pela primeira vez: $\eta_{2}=0,41$; parceiros: $\left.\eta_{2}=0,528\right)$ mostraram grande tamanho de efeito. 
Revista Ibero- Americana de Humanidades, Ciências e Educação- REASE

оPEN $\widehat{\text { Access }}$

\begin{tabular}{|c|c|c|c|c|c|c|}
\hline$\# \mathbf{N}$ & Data & Título & Autores & Periódico & Objetivos & Resultados \\
\hline 4 & 2017 & $\begin{array}{l}\text { Women's sense of coherence } \\
\text { and its association with early } \\
\text { weaning }\end{array}$ & $\begin{array}{l}\text { Fernando M. Cortelo, } \\
\text { Sérgio T.M. Marba, } \\
\text { Karine L. Cortellazzi, } \\
\text { Glaucia M M.B. } \\
\text { Ambrosano, Luciane } \\
\text { M. Guerra, Ana } \\
\text { Cláudia. Almeida, } \\
\text { Scott L. Tomar, } \\
\text { Margarete Ribeiro da } \\
\text { Silva e Rosana F. } \\
\text { Possobond. }\end{array}$ & $\begin{array}{l}\text { Jornal de } \\
\text { Pediatria }\end{array}$ & $\begin{array}{l}\text { Investigar se há associac, ão } \\
\text { entre níveis de senso de } \\
\text { coerência e desmame precoce. }\end{array}$ & $\begin{array}{l}\text { Os resultados apontaram que mães com maior senso de coerência possuem } 1,82 \text { vez } \\
\text { mais chance de manter o aleitamento por mais tempo }(\mathrm{p}=0,02) \text {. }\end{array}$ \\
\hline
\end{tabular}




\section{DISCUSSÃO}

O objetivo do presente estudo foi avaliar o senso de coerência (SOC) de mães em vulnerabilidade social e o estado nutricional de seus filhos, uma vez que o SOC está interligado à forma como essas mães lidam com situações estressantes, mantendo seus filhos saudáveis.

E, analisando a prática de aleitamento materno exclusivo, verificou-se que o baixo senso de coerência está associado ao desmame precoce, uma vez que essas mães nem sempre possuem recursos de enfrentamento ideal para as adversidades (CORBELO et al., 2017).

Isto é, os estressores intrínsecos ao desmame precoce, por exemplo, é o retorno ao trabalho, intercorrências mamárias e percepção de leite fraco, entre outros. Por isso, as mães com alto SOC, lidam de forma mais adequada, persistindo na amamentação (CORBELO et al., 2017).

Não só isso, mas estudos evidenciam a importância de ver amamentação não apenas como forma de alimentar a criança, mas também como uma aproximação entre mãe e filho e um desafio existencial para a lactante (GRANBERG; EKSTROM-BERGSTROM; BACKSTROM, 2020).

Neste sentido, a transição para a paternidade é uma das mudanças mais extrema que ocorrem na vida das pessoas, sendo uma oportunidade de crescimento ou uma experiência estressante, pois envolve ajustes fisiológicos, psicológicos e sociais. (BACKSTROM et al., 2018).

Além disso, um dos fatores positivos que contribui para o bem-estar dos casais durante a transição foi um forte senso de coerência familiar. O SOC familiar pode promover uma boa adaptação durante a transição para a paternidade (NGAI; CHAN, 2020).

Desse modo, estudos enfatizaram que para aumentar o senso de coerência familiar, era importante também passar tempo com a família e manter o equilíbrio trabalho-família (NGAI; CHAN, 2020). 
Ngai; Chan (2020) Salientam que, embora, existam mudanças na vida pessoal durante a maternidade, ao perceber a demanda do papel dos pais, os casais implantaram recursos próprios para enfrentar os desafios. Com isso, fornece base motivacional para o compromisso e devoção à família. Neste contexto, estudos evidenciam que além do apoio de membros da família, os casais buscaram aconselhamento e informações em redes sociais, amigos, colegas, profissionais de saúde, grupos de apoio religioso e grupos online de pais (NGAI; CHAN, 2020).

Além disso, Eli et al., (2016) descreve que o SOC é influenciado pelo status socioeconômico (SES). Este trabalho está de acordo com um estudo realizado no Japão que descobriu que o SOC ruim era comum em famílias com menor renda (MIZUTA et al.,2020).

No entanto, Speirs (2016) descreve que as famílias de baixa renda podem ser resilientes ao ambiente obesogênico, desde que tenha um forte senso de coerência familiar (FSOC) podendo ser capazes de reunir os recursos necessários.

Mais também, a influência dos aspectos sociodemográficos, especificamente a escolaridade materna no SOC é achado relevante, já que os níveis de SOC das mães aumentam quando relacionados à educação e diminuiu com um Índice de Massa Corpórea (IMC) maior (Eli et a., 2016).

Além disso, é importante observar que a maior escolaridade materna tem sido relacionada com o hábito frequente entre crianças e adolescentes de assistir TV, aumentando o comportamento sedentário (PIMENTA et al., 2020).

Granberg; Ekstrom-Bergstrom; Backstrom (2020) descreve em seu estudo, realizado com mães na Suécia que é improvável que fatores socioeconômicos possam explicar muito do que afeta a experiência duração da amamentação.

Nesse sentido, sabe-se que pessoas com forte senso de coerência tem boa percepção sobre sua saúde e melhor qualidade de vida, apresentam menos fadiga, depressão, solidão e ansiedade (CORBELO et a., 2017).

Em relação às práticas alimentares, Eli et al., (2016), verificou que mães com altos níveis de SOC e maior resiliência ao estresse, têm menor possibilidade de expressar preocupação com o peso excessivo de seus filhos ou a ingestão de alimentos não saudáveis. 
Portanto, tendo em vista que a preocupação com o peso da criança está ligada negativamente ao SOC e positivamente ligada à restrição; até $26 \%$ do efeito do SOC na restrição é mediado por preocupação (ELI et al., 2016).

Com isso, Eli et al., (2016), ao analisar o SOC e associar com o Questionário de Alimentação Infantil (CFQ) entre mães de pré-escolares na Suécia, identificou que mães com maior senso de coerência eram menos propensas a alimentação restritiva ou de pressão para comer.

Nesse contexto, a resiliência ao estresse pode reduzir a perspectiva, de as mães se envolverem em prática prejudicial, mesmo na presença de preocupação com o peso da criança (ELI et al., 2016).

Assim, os achados indicam que os programas voltados para educação nutricional e comunidades de menor renda devem incluir parâmetros relacionados ao SOC como parte do processo de avaliação, a fim de melhorar desenvolvimento e eficácia do programa (ELI et al., 2016).

Portanto, as pesquisas sobre obesidade infantil, que consideram o ambiente doméstico, concentram-se nas práticas parentais, e nas características demográficas. Além disso, o SOC familiar pode beneficiar os em vulnerabilidade e reduzir o impacto do ambiente obesogênio (SPEIRS et al., 2016).

Dessa maneira, foi possível observar que a busca para avaliar o SOC materno e estado nutricional de pré-escolares são escassos. Logo, se faz necessária a realização de pesquisa que avalie SOC de mães em vulnerabilidade social e estado nutricional de seus filhos, visando conhecer a realidade dessa população e criar subsídios para direcionar estratégias mais adequadas e conduzir futuras pesquisas na área.

Em síntese, essas razões apontadas poderiam justificar se o aumento do SOC pode promover comportamentos maternos saudáveis, limitar práticas parentais não saudáveis e, melhorar as estratégias de enfrentamento das adversidades.

\section{CONCLUSÃO}

O objetivo do presente estudo foi avaliar o senso de coerência (SOC) de mães em vulnerabilidade social e o estado nutricional de seus filhos.

Os achados enfatizam que mães com SOC aumentado (forte) podem ser mais resilientes frente às adversidades, (depressão, dificuldades na amamentação, os 
custos elevados de alimentos, a falta de espaço para recreação) e mesmo assim manter estratégias de enfrentamento adequadas.

Bem como, menos propensa a práticas alimentares que levem a obesidade infantil, além disso, possuem melhor capacidade de lidar com assuntos referentes ao peso de seus filhos.

Realizar a proposta de avaliar o SOC materno e o estado nutricional de préescolares é algo inovador e pouco estudado na literatura, este trabalho expande o campo de abordagem para este tema em questão.

\section{REFERÊNCIAS}

ANTONOVSKY, A. The structure and properties of the sense of coherence scale. Social Science \& Medicine.Israel vol. 36, n. 6, p. 725-733, 1993.

BACKSTROM, C. et al. Quality of couple relationship among first-time mothers and partners, during pregnancy and the first six months of parenthood. Sexual \& Reproductive Healthcare. Sweden.p. 56-64, Jul 2018. https://doi.org/ro.1016/j.srhc.2018.07.oor.

BAUER, F.G. et al. Future directions for the concept of salutogenesis: a position article. Health Promotion International. Inglaterra, p.I-9, jul 20I9. doi: I0.1093/heapro/daz057. Acesso em : 01-04-2021

CORBELO, M.F et al. Women's sense of coherence and its association with early weaning. J Pediatr. Campinas, SP, v.94,n.6,p.624-629,2017. ttps://doi.org/ro.1016/j.jped.2017.08.007

ELI, K. et al. Sorjonen K, Mokoena L ,Pietrobelli A , CE Flodmark , Faith MS et al. Associations between maternal sense of coherence and controlling feeding practices: The importance of resilience and support in families of preschoolers. Appetite. Sweden. v.105, p.134-143, 2016.

GRANBERG, A.; EKSTROM-BERGSTROM, A.; BACKSTROM, C. First-Time Mothers' Enjoyment of Breastfeeding Correlates with Duration of Breastfeeding, Sense of Coherence, and Parental Couple and Child Relation: A Longitudinal Swedish Cohort Stud. Nursing Research and Practice.v.2020, p.I-I3, Jun 2020, https://doi.org/10.1155/2020/8194389

MIZUTA, A. et al. O. Does the Association between Guardians' Sense of Coherence and their Children's Untreated Caries Differ According to Socioeconomic Status? Int. J. Environ. Res. Public. Health. Japão.v.I7,p. oI-I2,mar., 2020. 
NGAI, W.F.; CHAN, S.P. Perception of family sense of coherence during parental transition: A qualitative study. Journal of Health Psychology .Hong Kong, v.oo, n.o, p.I-I5,2020. DOI: I0.1177/1359105320914062. PIMENTA, F.M.V.et al. Antropometria e insegurança alimentar de adolescentes, inscritos e não inscritos no Programa Bolsa Família, no sudeste brasileiro. Braz. J. of Develop, Curitiba, v. 6, n. Io, p. 77160-77183, o9 oct. 2020, DOI: 10.34117/bjdv6nio-225.

SPEIRS, K. E. et al. Is Family Sense of Coherence a Protective Factor against the Obesogenic Environment? Appetite. USA.v.99, p. 268-276, jan 2016. 